\title{
Attention-deficit/hyperactivity disorder: variation by socio-economic deprivation
}

Vibhore Prasad ${ }^{1}$, Joe West ${ }^{2}$, Denise Kendrick ${ }^{3}$, Kapil Sayal ${ }^{4}$

${ }^{1}$ School of Population Sciences \& Health Services Research, King's College London, London, SE1 1UL, UK

Vibhore Prasad

NIHR Academic Clinical Lecturer in General Practice

${ }^{2}$ School of Medicine, Clinical Sciences Building 2, Nottingham City Hospital, University of Nottingham, Nottingham, NG5 1PB, UK

Joe West

Professor of Epidemiology

${ }^{3}$ School of Medicine, Tower Building, University of Nottingham, Nottingham, NG7 2RD, UK Denise Kendrick

Professor of Primary Care Research

${ }^{4}$ School of Medicine, Developmental Psychiatry, E Floor, South Block, Queen's Medical

Centre, University of Nottingham, Nottingham, NG7 2UH, UK

Kapil Sayal

Professor of Child and Adolescent Psychiatry

Correspondence to: V Prasad vibhore.prasad@kcl.ac.uk Telephone 07960-865339

Word count 1219 words 


\section{What is known about this topic}

Attention-deficit/hyperactivity disorder (ADHD) affects 3 to $5 \%$ of children and young people in the community in the United Kingdom.

There is a discrepancy between the community prevalence of ADHD and the clinically recorded prevalence, which is less than $1 \%$.

Estimates of how the clinically recorded prevalence of ADHD varies by deprivation and region are lacking.

\section{What this study adds}

The clinically recorded prevalence of ADHD in children and young people was twice as high in the most compared to the least deprived areas.

The greatest inequality in recorded prevalence of ADHD was in the East of England and the least inequality was in London.

There is a greater need for health and educational services for children with ADHD in more disadvantaged areas. 


\section{Acknowledgements}

This study was supported by a National Institute for Health Research (NIHR) grant, DRF2011-04-116. Dr Prasad reported having received research grant support administered via the University of Nottingham from the NIHR Doctoral Research Fellowship scheme. During the period of the NIHR award for VP, JW was supported by a University of Nottingham/Nottingham University Hospitals National Health Service (NHS) Senior Clinical Research Fellowship. There were no other financial relationships with any organisations that might have an interest in the submitted work. The authors have no conflicts of interest to disclose.

This article/paper/report presents independent research funded by the NIHR. The views expressed are those of the author(s) and not necessarily those of the NHS, the NIHR or the Department of Health.

\section{Competing Interest}

None declared 


\section{Abstract}

Background In England, there is a discrepancy between the prevalence of Attentiondeficit/hyperactivity disorder (ADHD) ascertained from medical records and community surveys. There is also a lack of data on variation in recorded prevalence by deprivation and geographical region; information that is important for service development and commissioning.

Methods Cohort study using data from the Clinical Practice Research Datalink comprising 5,196 children and young people aged 3-17 years with ADHD and 490,016 without, in 2012.

Results In 2012, the recorded prevalence (95\%Cl) of ADHD was $1.06(1.03-1.09) \%$. Prevalence in the most deprived areas was double that of the least deprived areas (prevalence rate ratio (PRR) $2.58(2.36-2.83))$, with a linear trend from least to most deprived areas across all regions in England.

Conclusions The low prevalence of ADHD in medical records may indicate considerable under-diagnosis. Higher rates in more disadvantaged areas indicates greater need for services in those areas. 


\section{Introduction}

The community prevalence of attention-deficit/hyperactivity disorder (ADHD) is $3-5 \%$ in

England ${ }^{1}$. However, the clinically recorded prevalence is much lower $(<1 \%)^{23}$. Estimates of how this varies by deprivation and region are lacking. This cohort study aims to address this gap and inform development of health and education services.

\section{Methods}

Data source

We used the General Practice (GP) medical records linked to hospital episodes statistics (HES) data from the Clinical Practice Research Datalink (CPRD) from 370 GP practices, which represents approximately $4 \%$ of the UK population ${ }^{4}$. These data are broadly representative of the UK population and contain information on consultations with GPS, hospital admissions, diagnoses and prescriptions ${ }^{4}$.

Defining the population with ADHD

We extracted medical records for children and young people (CYP) aged 3-17 years registered before June 2013 with at least one diagnosis code or prescription for ADHD. We took the latest of the date of: third birthday; diagnosis; registration with the practice (in CYP diagnosed before registration); or $1^{\text {st }}$ January 1998 (the first full year of the CPRD-HES link) as the date when ADHD was first known to the GP. We took the earliest of the date when the: CYP left the practice or died; practice stopped participating in the CPRD; CYP turned 18 years; or $31^{\text {st }}$ December 2012 (the last complete year that CPRD-HES linked data were available), as the last date of follow-up.

Estimating prevalence - numerators 
To calculate the frequency of recorded ADHD in 2012, CYP were required to have received the diagnosis on or prior to $1^{\text {st }}$ July and to be registered with the GP on $1^{\text {st }}$ July. The number of CYP known to have ADHD by $1^{\text {st }}$ July in 2012 was counted overall and by: age, sex, strategic health authority region and social deprivation quintile (English index of multiple deprivation (IMD) score 2010, at lower super output area level, based on home postcode). The IMD score comprises seven domains: income, employment, health and disability, barriers to housing and services, living environment and crime.

\section{Denominators}

We extracted medical records from the CPRD for CYP registered before $31^{\text {st }}$ December 2012. CYP who were: aged from 3-17 years old; registered with the practice and alive, between $1^{\text {st }}$ January 2012 and $31^{\text {st }}$ December 2012 were counted in the denominator. The number of CYP registered in the CPRD on $1^{\text {st }}$ July in 2012 was counted overall and by age, sex, region and deprivation).

Prevalence estimates

Prevalence rates for 2012 were estimated assuming a Poisson distribution and described overall, by sex, age, region and deprivation. We assessed whether prevalence by age varied by sex and whether deprivation gradients varied by region by adding interaction terms to the model assessing significance using a likelihood ratio test (LRT). 
Sensitivity analyses and subgroup analyses

To explore how altering the definition of ADHD affects estimates of recorded prevalence, we described subgroups reflecting at least one drug code or at least one diagnosis code; at least two drug codes and at least two diagnosis codes; at least one drug code; no drug codes.

Ethics

Approval was obtained from CPRD's independent scientific advisory committee (ISAC) (Protocol reference 12_128R). 


\section{Results}

There were 5,196 CYP with ADHD and 490,016 without (Table 1). In 2012, the recorded prevalence $(95 \% \mathrm{Cl})$ of ADHD was $1.06(1.03-1.09) \%$. Boys had a five-fold higher prevalence than girls ( $1.74 \%$ vs. $0.35 \%$, PRR $4.98(4.62-5.36) \%)$. The prevalence was highest in 15 to 17 -year-olds (1.91 (1.82-1.99) \%) and lowest in 3 to 4-year-olds (0.01 $(0.002-0.02) \%)$. The relationship between prevalence and age did not differ significantly by sex (LRT $p=0.09$ ). Prevalence rates increased with increasing deprivation, being two-fold higher in CYP from the most compared with the least deprived areas $(1.38 \%$ vs $0.73 \%$, PRR 2.58 (2.36-2.83) \%; test for linear trend $p<0.001)$. There was considerable geographic variation, with higher prevalence in the South East and East regions, compared to Yorkshire and Humber (1.55 \% and $1.34 \%$ vs. $0.56 \%$, PRR 3.13 (2.46-3.99) and 2.80 (2.19-2.00)), respectively. Varying definitions of ADHD did not alter prevalence patterns by sex, age, deprivation or region (online supplementary table). 
Table 1: The recorded prevalence, crude and adjusted prevalence rate ratio (PRR) of ADHD in 2012

\begin{tabular}{|c|c|c|c|c|c|c|c|c|c|c|c|c|}
\hline \multirow{2}{*}{$\begin{array}{l}\text { Characteristic } \\
\text { Overall }\end{array}$} & \multirow{2}{*}{$\begin{array}{r}{ }^{\mathrm{f} A D H D} \\
5,196\end{array}$} & \multirow{2}{*}{\begin{tabular}{|l|} 
fNo ADHD \\
490,016
\end{tabular}} & \multirow{2}{*}{$\begin{array}{r}\text { Prevalence \% } \\
1.06\end{array}$} & \multicolumn{2}{|c|}{$(95 \% \mathrm{Cl})$} & \multirow[t]{2}{*}{ PRR } & \multicolumn{2}{|c|}{$95 \% \mathrm{Cl}$} & \multirow[t]{2}{*}{$\begin{array}{l}{ }^{\$} \text { Adjusted } \\
\text { PRR }\end{array}$} & \multicolumn{2}{|c|}{$95 \% \mathrm{Cl}$} & P-value \\
\hline & & & & (1.03 & $-1.09)$ & & - & & & - & & 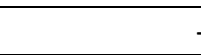 \\
\hline Female & 841 & 240,034 & 0.35 & $(0.33$ & $-0.37)$ & & 1 & & & 1 & & $p<0.001^{\&}$ \\
\hline Male & 4,355 & 249,982 & 1.74 & $(1.69$ & $-1.79)$ & 4.97 & (4.62 & $-5.35)$ & 4.98 & $(4.62$ & $-5.36)$ & \\
\hline Age group & $\$ \$$ & 69,713 & 0.01 & $(0.002$ & $-0.02)$ & & 1 & & & 1 & & $p<0.001^{\#}$ \\
\hline $5-9$ & 830 & 163,834 & 0.51 & (0.47 & $-0.54)$ & 70.6 & $(29.3$ & $-170.1)$ & 71.7 & $(29.8$ & $-173)$ & \\
\hline $10-14$ & 2,467 & 157,090 & 1.57 & $(1.51$ & $-1.63)$ & 219 & (91.1 & $-527)$ & 226 & (93.9 & $-543)$ & \\
\hline $15-17$ & 1,894 & 99,379 & 1.91 & $(1.82$ & $-1.99)$ & 266 & $(110$ & $-639)$ & 275 & (114 & $-662)$ & \\
\hline \multirow{2}{*}{ DeprivationLeast deprived } & 870 & 118,422 & 0.73 & $(0.69$ & $-1.79)$ & & 1 & & & 1 & & $p<0.001^{\#}$ \\
\hline & 926 & 99,463 & 0.93 & $(0.87$ & $-0.99)$ & 1.27 & (1.16 & $-1.39)$ & 1.36 & $(1.23$ & $-1.49)$ & \\
\hline Medium deprivation & 908 & 86,374 & 1.05 & $(0.98$ & $-1.12)$ & 1.43 & $(1.30$ & $-1.57)$ & 1.58 & $(1.44$ & $-1.74)$ & \\
\hline 2nd most deprived & 1,169 & 90,230 & 1.30 & (1.22 & $-1.37)$ & 1.76 & (1.62 & $-1.93)$ & 2.10 & (1.92 & $-2.30)$ & \\
\hline \multirow{2}{*}{$\begin{array}{r}\text { Most deprived } \\
\text { Data missing }\end{array}$} & 1,211 & 87,564 & 1.38 & $(1.31$ & $-1.46)$ & 1.88 & $(1.73$ & $-2.05)$ & 2.58 & $(2.36$ & $-2.83)$ & \\
\hline & 112 & 7,963 & 1.41 & $(1.16$ & $-1.69)$ & 1.91 & $(1.57$ & $-2.33)$ & 2.53 & $(2.08$ & $-3.08)$ & \\
\hline East Midlands & 79 & 7,480 & 1.06 & $(0.84$ & $-1.32)$ & 1.90 & (1.38 & $-2.62)$ & 1.95 & $(1.42$ & $-2.69)$ & $p<0.001^{\&}$ \\
\hline East of England & 698 & 51,995 & 1.34 & (1.24 & $-1.45)$ & 2.41 & (1.89 & $-3.08)$ & 2.80 & $(2.19$ & $-2.00)$ & \\
\hline London & 630 & 83,266 & 0.76 & $(0.70$ & $-0.82)$ & 1.36 & (1.06 & $-1.74)$ & 1.36 & (1.06 & $-1.74)$ & \\
\hline North East & 111 & 11,165 & 0.99 & $(0.82$ & $-1.20)$ & 1.79 & (1.33 & $-2.41)$ & 1.54 & (1.15 & $-2.08)$ & \\
\hline North West & 717 & 80,541 & 0.89 & $(0.83$ & $-0.96)$ & 1.60 & (1.25 & $-2.04)$ & 1.48 & (1.16 & $-1.89)$ & \\
\hline South Central & 860 & 67,269 & 1.28 & (1.19 & $-1.37)$ & 2.30 & (1.80 & $-2.93)$ & 2.70 & $(2.12$ & $-3.44)$ & \\
\hline South East Coast & 1,046 & 67,691 & 1.55 & (1.45 & $-1.64)$ & 2.78 & (2.18 & $-3.53)$ & 3.13 & $(2.46$ & $-3.99)$ & \\
\hline South West & 534 & 54,875 & 0.97 & $(0.89$ & $-1.06)$ & 1.75 & (1.37 & $-2.24)$ & 1.79 & (1.39 & $-2.29)$ & \\
\hline West Midlands & 450 & 52,969 & 0.85 & $(0.77$ & $-0.93)$ & 1.53 & (1.19 & $-1.96)$ & 1.56 & $(1.21$ & $-2.00)$ & \\
\hline Yorkshire \& Humber & 71 & 12,765 & 0.56 & $(0.43$ & $-0.70)$ & & 1 & & & 1 & & \\
\hline
\end{tabular}

${ }^{f}$ Number of children and young people in 2012

$\$$ Adjusted for the other variables in the table

\&P value for likelihood ratio test comparing the adjusted model with the variable to a model without

${ }^{\$ \$}$ Frequencies less than 10 not displayed to protect anonymity

\#P value for test for linear trend adjusting for other variables in the table 
The social gradient in ADHD prevalence was evident within all regions and also varied significantly between regions (Figure 1 , test for interaction $\mathrm{p}<0.001$ ). The steepest social gradient (comparing most to least deprived areas) was in the East of England (PRR 3.43 (2.70-4.37)) and the smallest gradient in London (PRR 1.38 (1.04-1.83)). 


\section{Discussion}

The recorded prevalence of ADHD in CYP (1.06\%) in 2012 was considerably lower than community prevalence estimates ${ }^{1}$. Recorded prevalence estimates worldwide vary from $0.06 \%$ to $13 \%$, with higher estimates originating from the USA ${ }^{5}$. The prevalence was double in the most compared to the least deprived areas with a linear trend across all regions in England. The greatest inequality was within the East of England region and the least within London.

Our low prevalence estimate suggests that there are many CYP in the population with undiagnosed ADHD. This is supported by findings from the British Child and Adolescent Mental Health Survey ${ }^{1}$, which reported ADHD prevalence amongst $5-15$ year-olds of $2.23 \%$. Under-diagnosis is important as it precludes receipt of appropriate child and parental support, educational support and behavioural and pharmacological treatment for ADHD. This may be particularly relevant as children transition from primary to secondary school and independent learning is increasingly required. As recorded prevalence of ADHD increases with age, and we included children aged under five, this may partly explain our low prevalence estimate. It is also possible that secondary care ADHD diagnoses are not being recorded in primary care records. However, systematic reviews demonstrate accurate recording of secondary care diagnoses in primary care records and high validity across a wide range of diagnoses, so this is unlikely to explain much of the difference in prevalence rates $^{67}$.

Worldwide studies of community prevalence of ADHD suggest CYP from disadvantaged families are 1.5 to 4 times more likely to have ADHD symptoms compared to those from more advantaged families ${ }^{8}$. This is consistent with Millennium Cohort Study (MCS) findings of strong associations between multiple measures of socio-economic disadvantage and parent-reported diagnosed prevalence of $\mathrm{ADHD}^{9}$. This may represent true differences in the 
prevalence of ADHD, differential symptom reporting or differential access to services to diagnose and treat ADHD. The MCS found similar associations between parent or teacher reported ADHD symptoms and socio-economic disadvantage, suggesting that clinical labelling bias does not explain the social gradient ${ }^{9}$. Potential explanations for the social gradient, include differential exposures to multiple material, psychosocial and environmental risk factors before or around the time of birth or in childhood, as well as genetic or developmental contributions ${ }^{9}$. Genetic susceptibility may also play a role, with some individuals being more susceptible to adverse, or supportive, environmental exposures ${ }^{10}$.

Our findings suggest greater need for health and educational services for CYP with ADHD in more disadvantaged areas and can inform the development and commissioning of appropriate services, with our figures being understood as conservative estimates. Future research is needed to explore under-diagnosis or under-recording of ADHD in CYP and mechanisms by which socio-economic disadvantage impacts on ADHD prevalence. 


\section{References}

1. Ford T, Goodman R, Meltzer H. The British Child and Adolescent Mental Health Survey 1999: The Prevalence of DSM-IV Disorders. Journal of the American Academy of Child \& Adolescent Psychiatry 2003;42(10):1203-11. doi: http://dx.doi.org/10.1097/00004583-200310000-00011

2. Hsia Y, Maclennan K. Rise in psychotropic drug prescribing in children and adolescents during 1992-2001: a population-based study in the UK. European Journal of Epidemiology 2009;24(4):211-16.

3. McCarthy S, Wilton L, Murray M, et al. The epidemiology of pharmacologically treated attention deficit hyperactivity disorder (ADHD) in children, adolescents and adults in UK primary care. BMC pediatrics 2012;12(1):78.

4. Herrett E, Gallagher AM, Bhaskaran K, et al. Data Resource Profile: Clinical Practice Research Datalink (CPRD). International Journal of Epidemiology 2015;44(3):827-36. doi: 10.1093/ije/dyv098

5. Sayal K, Prasad V, Daley D, et al. ADHD in children and young people: prevalence, care pathways, and service provision. Lancet Psychiatry 2018;5(2):175-86. doi: 10.1016/S2215-0366(17)30167-0 [published Online First: 2017/10/17]

6. Herrett E, Thomas SL, Schoonen WM, et al. Validation and validity of diagnoses in the General Practice Research Database: a systematic review. Br J Clin Pharmacol 2010;69(1):4-14. doi: 10.1111/j.1365-2125.2009.03537.x

7. Khan NF, Harrison SE, Rose PW. Validity of diagnostic coding within the General Practice Research Database: a systematic review. Br J Gen Pract 2010;60(572):e128-36. doi: 10.3399/bjgp10X483562 [doi] [published Online First: 2010/03/06]

8. Willcutt EG. The prevalence of DSM-IV attention-deficit/hyperactivity disorder: a metaanalytic review. Neurotherapeutics 2012;9(3):490-99.

9. Russell G, Ford T, Rosenberg R, et al. The association of attention deficit hyperactivity disorder with socioeconomic disadvantage: alternative explanations and evidence. Journal of Child Psychology \& Psychiatry \& Allied Disciplines 2014;55(5):436-45. doi: http://dx.doi.org/10.1111/jcpp.12170

10. Belsky J, Pluess M. Beyond diathesis stress: Differential susceptibility to environmental influences. Psychological Bulletin 2009;135(6):885-908. doi: 10.1037/a0017376 
Figure 1 Prevalence of ADHD by area-level deprivation for each region 